\title{
Serious Adverse Events Associated with Off-Label Use of Azithromycin or Fentanyl in Children in Intensive Care Units: A Retrospective Chart Review
}

\author{
Kazeem A. Oshikoya ${ }^{1} \cdot$ Gerold T. Wharton ${ }^{2}$. Debbie Avant ${ }^{2} \cdot$ Sara L. Van Driest ${ }^{1} \cdot$ Norman E. Fenn III $^{3}$.

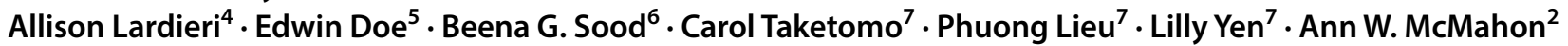

Published online: 28 November 2018

(C) This is a U.S. government work and its text is not subject to copyright protection in the United States; however, its text may be subject to foreign copyright protection 2018

\begin{abstract}
Objectives Half of prescription drugs commonly given to children lack product labeling on pediatric safety, efficacy, and dosing. Two drugs most widely used off-label in pediatrics are azithromycin and fentanyl. We sought to determine the risk of serious adverse events (SAEs) when oral azithromycin or intravenous/intramuscular fentanyl are used off-label compared to on-label in pediatric intensive care units (ICUs).

Study Design Six pediatric hospitals participated in a retrospective chart review of patients administered oral azithromycin $(n=241)$ or intravenous/intramuscular fentanyl $(n=367)$ between January 5, 2013 and December 26, 2014. Outcomes were SAEs by drug and labeling status: off-label compared to on-label by Food and Drug Administration (FDA)-approved age and/or indication. Statistical analysis was performed using logistic regression to estimate odds ratios (ORs) and Cox regression to estimate hazard ratios (HRs).

Results Twenty-one (9\%) children receiving azithromycin experienced SAEs. Off-label use of azithromycin was not associated with a higher risk of SAE (OR 0.87, 95\% CI 0.27-2.71, $p=0.81$ ). Ninety-five (26\%) children receiving fentanyl experienced SAEs. Fentanyl off-label use by both age and indication was not associated with a higher risk of overall SAEs compared to on-label use (OR 1.99, 95\% CI 0.94-4.19, $p=0.07$ ). However, the risk of the SAE respiratory depression was significantly greater when fentanyl was used off-label by both age and indication (OR 5.05, 95\% CI 1.08-23.56, $p=0.044$ ). Results based on HRs were similar.

Conclusions Azithromycin off-label use in pediatric ICUs does not appear to be associated with an increased risk of SAEs. Off-label use of fentanyl appears to be more frequently associated with respiratory depression when used off-label by both age and indication in pediatric ICUs. Prospective studies should be undertaken to assess the safety and efficacy of fentanyl in the pediatric population so that data can be added to the FDA labeling.
\end{abstract}

Kazeem A. Oshikoya and Gerold T. Wharton contributed equally.

Electronic supplementary material The online version of this article (https://doi.org/10.1007/s40272-018-0318-9) contains supplementary material, which is available to authorized users.

Ann W. McMahon

Ann.mcmahon@fda.hhs.gov

1 Vanderbilt University School of Medicine, Nashville, TN, USA

2 Office of Pediatric Therapeutics, Office of the Commissioner, Food and Drug Administration, 10903 New Hampshire Avenue, Building 32, Room 5158, Silver Spring, MD 20993,

3 Children's National Health System, Washington, DC, USA

4 University of Maryland Children's Hospital, Baltimore, MD, USA

5 INOVA Children's Hospital, Falls Church, VA, USA

6 Children's Hospital of Michigan, Detroit, MI, USA

7 Children's Hospital Los Angeles, Los Angeles, CA, USA USA 


\section{Key Points}

Off-label use of azithromycin and fentanyl is common among children admitted to intensive care units.

Off-label use compared to on-label use of fentanyl in children in intensive care units may be associated with some serious adverse events.

Practitioners taking care of children using fentanyl offlabel in intensive care units may want to watch these children carefully and monitor, especially for respiratory events, as is recommended in the black box warning in the fentanyl labeling. At least until more information is available about the safety of off-label fentanyl use in children, practitioners using fentanyl in this population should be aware of whether the drug is being used on- or off-label, and be vigilant.

\section{Introduction}

Half of the prescription medications commonly given to children lack information in the product labeling regarding pediatric safety, efficacy and dosing [1]. Consequently, many drugs are used off-label in children [2, 3]. Among the various drug classes often prescribed off-label for children, antimicrobial agents predominate, followed by analgesics (with or without sedation), bronchodilators, topical agents, sex hormones, cardiovascular drugs, and psychotropic drugs [4]. Studies that have explored whether off-label drug use in pediatrics is associated with adverse outcomes have generally used the outcome terminology of adverse drug event (ADE), which is defined as "any adverse event associated with the use of a drug in humans, whether or not considered drug related" [5]. In our study, we focus on serious adverse events (SAEs) resulting in death, a life-threatening condition, hospitalization or prolongation of hospitalization, persistent or significant disability or incapacity, congenital anomaly, or other event requiring intervention [5] as associated with off-label drug use in children.

A recent study of 694 individuals from the United Kingdom indicated that unlicensed and off-label drug use for pediatric inpatients were more likely than the use of authorized drugs to produce ADEs within a cohort of hospitalized children [6]. However, studies that specifically evaluate the incidence of SAEs among hospitalized children are lacking.

Azithromycin and fentanyl are among the most frequently used drugs in neonatal intensive care units (NICUs) and pediatric intensive care units (PICUs) in the USA [7, 8]. They represent two groups of drugs, antibiotics and analgesics, which are most commonly used off-label in pediatrics
$[2,3]$. These two drugs are Food and Drug Administration (FDA) - approved for use in restricted pediatric age groups and for specific indications. However, neither oral azithromycin nor intravenous (IV)/intramuscular (IM) fentanyl has been studied under the Best Pharmaceuticals for Children Act (BPCA) or the Pediatric Research Equity Act (PREA), two legislative programs that the FDA utilizes for clinical studies to be conducted in pediatrics. Both of these drugs were labeled before this legislation took effect. A large study involving an audit of the Pediatric Health Information System (PHIS) database for pediatric inpatients from 36 children's hospitals in the USA showed that azithromycin and fentanyl were used off-label by $89 \%$ and $87 \%$ of the studied cohort, respectively [9].

Azithromycin is FDA - approved for acute bacterial sinusitis and community acquired pneumonia in adults and pediatric patients who are $\geq 6$ months old [10]. There are limited safety data on azithromycin use in children $<6$ months old, and it has not been approved for use in this age group. However, it is used off-label for a variety of reasons, including the treatment and chemoprophylaxis of pertussis [11].

Fentanyl is approved for use in children $\geq 2$ years old for analgesic action of short duration during the anesthetic periods, premedication, induction and maintenance, and in the immediate postoperative period (recovery room) as the need arises (PRN) [12]. Fentanyl is not approved for use outside the perioperative period for pain or sedation [12]. However, fentanyl, like other sedating drugs, is used off-label in children outside the operating room [13]. A recent Canadian study classified all drugs given to children $<18$ years of age in a single day at a tertiary care children's hospital as on- or off-label. In this study, 38\% of drugs were given off-label and fentanyl was among the twenty most frequently given off-label drugs [14]. All 17 prescriptions for fentanyl were prescribed off-label, with $88 \%$ off-label by age and $12 \%$ offlabel due to unapproved dosing frequency [14].

There are a few studies documenting the safety of drugs that are frequently administered to children in the ICU [15-17]. However, no published study has documented the correlation of SAEs with on- or off-label use of azithromycin or fentanyl in children. The primary objective of this study was to determine whether SAEs are more likely to occur with off-label use of oral azithromycin or IV/IM fentanyl as compared to on-label use in children admitted to the PICU, NICU, or other ICUs of six pediatric hospitals in the USA.

\section{Methods}

\subsection{Study Design}

The study was performed using the electronic health records (EHRs) from six pediatric hospitals in the USA: Inova 
Fairfax Hospital, Fairfax, VA; Children's National Health System, Washington, DC; University of Maryland Children's Hospital, Baltimore, MD; Children's Hospital of Michigan, Detroit, MI; Children's Hospital Los Angeles, Los Angeles, CA; and Vanderbilt University Medical Center, Nashville, TN. The six pediatric hospitals contributed 241 patients that had used azithromycin and 367 patients that had used fentanyl (Table 1). The institutional review board for each participating hospital approved the study protocol.

Pharmacists at the Children's Hospital Los Angeles identified 135 drugs that are most frequently used in their PICU, NICU, or other ICUs. An FDA pharmacist reviewed the labeling for each of the 135 drugs. Twenty-four of the 135 drugs were eligible for further consideration because each of the 24 drugs met criteria 1 and 2 listed below. Drugs not approved for pediatric use and drugs approved for the entire pediatric population were not eligible for selection. Two drugs were selected (azithromycin and fentanyl) based on the following criteria: (1) the drug was on the list of drugs most frequently used in the PICU, NICU, or other ICUs $(n=135)$, (2) the drug was approved by the FDA for use for a particular indication in an age subset of the pediatric population $(n=24)$, and (3) the drug was either an antibiotic $(n=9)$ or a sedative/analgesic $(n=2)$, since these drugs are among the most frequently used off-label in children [7, 8]. One example each of an eligible antibiotic and sedative/ analgesic were chosen for this study.

Pharmacists or medical doctors trained in data extraction from each hospital searched EHRs for all the data extracted in the study and to identify patients meeting the inclusion criteria: in a NICU, PICU, or other ICU at the time of study drug administration, administered oral azithromycin $(n=241)$ or IV/IM fentanyl $(n=367)$ between January 5, 2013 and December 26, 2014, and $\leq 20$ years of age at admission. Exclusion criteria included the following: insufficient data to determine exposure(s) or outcome and having instances of both off-label and on-label exposures for either drug.

\subsection{Sample Size Determination}

The study was composed of a convenience sample, consecutively selected, who met the study inclusion criteria at the six children's hospitals.

\subsection{Case Definition and Identification}

Azithromycin or fentanyl use during ICU admission was categorized as "off-label by age and/or indication" or "onlabel." We defined on-label use of azithromycin as the oral administration of the drug for community acquired pneumonia or sinusitis in pediatric patients $\geq 6$ months old. We defined on-label use of fentanyl as the IV or IM administration of the drug for use in children $\geq 2$ years for analgesic action of short duration during the anesthetic periods, premedication, induction and maintenance, and in the immediate postoperative period $(\leq 4 \mathrm{~h})$ as the need arises. Off-label use of the drugs refers to any use outside the recommended age or indication. Our study cohort included children $\leq 20$ years of age to be inclusive of those patients who might have pediatric phenotypes despite this cut-off not being consistent with the FDA definitions of "pediatrics" with an upper age limit of 16 years for prescription drugs [18].

\subsection{Primary Outcome and Potential Confounders}

The primary outcome in our study was the presence of any SAE following exposure to azithromycin or fentanyl, which included clinically diagnosed SAEs (those reported by the patient or their parent/guardian or those discovered by clinicians after physical examination) and those detected from laboratory or diagnostic investigations. Investigators manually reviewed the EHR of each case. SAEs were defined as any adverse drug experience occurring at any dose that results in death, a life-threatening condition, hospitalization or prolongation of hospitalization, persistent or significant

Table 1 Pediatric hospital beds and contributions of patients to study

\begin{tabular}{lllcccc}
\hline Hospital & $\begin{array}{l}\text { Number beds pediatric } \\
\text { hospital }\end{array}$ & $\begin{array}{l}\text { Number beds } \\
\text { NICU }\end{array}$ & $\begin{array}{l}\text { Number beds } \\
\text { PICU }\end{array}$ & $\begin{array}{l}\text { Number beds other } \\
\text { ICU }\end{array}$ & $\begin{array}{l}\text { Azithromycin patients } \\
\text { contributed }\end{array}$ & $\begin{array}{l}\text { Fentanyl } \\
\text { patients con- } \\
\text { tributed }\end{array}$ \\
\hline 1 & 145 & 52 & 19 & 0 & 57 & 11 \\
2 & 254 & 39 & 48 & 24 & 43 & 50 \\
3 & 223 & 108 & 26 & 22 & 55 & 45 \\
4 & 267 & 96 & 42 & 25 & 42 & 52 \\
5 & 316 & 54 & 46 & 0 & 40 & 149 \\
6 & 361 & 58 & 24 & 71 & 241 & 367 \\
\hline Total & 1566 & 407 & 205 & & \\
\hline
\end{tabular}

$N I C U$ neonatal intensive care unit, $P I C U$ pediatric intensive care unit 
disability or incapacity, congenital anomaly, or other event requiring intervention [5]. Specific SAEs from the drug label were included on the report form, and free text fields were also included for the chart abstractor to write in additional details. The abstractors were instructed to include all those events resulting in the regulatory definition of SAE as provided above. For this study, investigators determined the incidence of these outcomes following an administration of oral azithromycin or IV/IM fentanyl. For our study, SAEs were eligible events for analysis if they took place while the patient was taking the study drug or during metabolic clearance of the study drug after it was discontinued, which was calculated as a lag of 24 days for azithromycin and 2 days for fentanyl [19].

Causality between drug and SAE was not assessed. Each investigator worked independently on SAE assessment. There was no formal adjudication process.

Potential confounders for the primary outcome (any SAE) were determined a priori and collected through manual review of the EHR. These variables included demographic data (age, sex, race, ethnicity), drug exposure data (dosing data described below, concurrent analgesic or antiemetic exposure for azithromycin, concurrent central nervous system depressant or vasopressor exposure for fentanyl, total concomitant medications), and location (PICU vs. NICU vs. other). In addition, the comorbid conditions at the time of drug exposure for everyone were collected. Dichotomous variables for comorbidity within each of 17 potential diagnostic classes (infectious and parasitic disease, respiratory disease, perinatal disease, etc.) were defined for everyone, as well as a total comorbidities variable representing the number of diagnostic classes affected for everyone.

\subsection{Dosing Definitions}

Dosing variables for azithromycin and fentanyl were collected from the medication administration record section of the EHR. For both drugs of interest, dosing regimens often changed throughout hospitalization. Specific start dates and end dates for each unique regimen were noted. If not already done so in the EHR, dosing was adjusted to weight-based dosing in all patients ( $\mathrm{mg} / \mathrm{kg}$ for azithromycin and $\mathrm{mcg} / \mathrm{kg}$ for fentanyl). For azithromycin and fentanyl, two dosing variables were defined post-analysis: maximum daily dose and cumulative dose. Maximum daily dose was calculated by taking the maximum value from a series of values of total drug administered to a patient in a 24-h period. For cumulative dose, a sum was calculated accounting for all drug regimens given during the patient's entire hospitalization. For fentanyl, dosing regimens were noted to include continuous IV infusions and intermittent doses with many noting PRN administration. As the exact dosing was not known for those administered the drug PRN, we did not include fentanyl dosing variables in our primary analysis. However, a sensitivity analysis was performed to examine the impact of dose on the outcome of SAE in the subset of fentanyl patients who had no PRN doses.

\subsection{Statistical Analysis}

Regression analysis was used to determine whether off-label use influenced the probability of any SAE reported while the patient was taking the study drug or during metabolic clearance of the study drug after it was discontinued, with azithromycin and fentanyl datasets analyzed separately. The primary dependent variable for our regression models was the Boolean of whether the patient experienced any SAE during drug administration, with a lag period for metabolic clearance, which was estimated to be 24 days for azithromycin and 2 days for fentanyl. We analyzed the risk of respiratory depression as its own dependent variable in a separate analysis in the fentanyl cohort, as this was the most common SAE reported. Since the most common SAE for azithromycin (diarrhea) was experienced only by patients with onlabel use, a similar analysis was not done for azithromycin. We used logistical, or logit, binary regression models with odds ratios (ORs) reported and robust standard errors. In addition, we used Cox proportional hazard models to determine if the length of drug administration affected the risk of any SAE. Analyses and graphics were performed with Stata 13.1 (College Station, TX) including Pearson's correlation matrices, summary statistics, and logistical and Cox regression models. Any association was statistically significant at $p<0.05$.

The primary independent variable was whether the patient was administered the drug off-label. Off-label use was subdivided when the sample size was sufficiently large into three different, non-overlapping categories: (1) off-label by age only, (2) off-label by indication only, and (3) off-label by both age and indication, i.e., the drug was administered to a patient who is simultaneously outside the age group in the drug labeling and for an indication not in the drug labeling. Other right hand side variables, or confounders, were deemed eligible for individual regressions if they were correlated at an absolute value of $\geq 0.1$ (Pearson's correlation coefficient, $r$ ) with both the primary dependent variable, risk of having any SAE, and off-label administration. Eligible confounders are included in the regression models with their $p$ values reported. Confounders were omitted if they were deemed endogenous to the model or were collinear with other confounders at an absolute value of $\geq 0.9$ (Pearson's correlation coefficient, $r$ ). For each regression analysis, individuals were excluded if they had missing data for any of the included confounders.

Three sensitivity analyses were performed. The first two are similar to the primary regression models for 
azithromycin and fentanyl, but change the cut-off for eligible confounders to an absolute value of $\geq 0.05$ (Pearson's correlation coefficient, $r$ ). The third sensitivity analysis retains the broader inclusion criteria for confounders, but also includes dosing variables for fentanyl. This analysis includes fewer patients as it omits those with any PRN dosing.

\section{Results}

The six participating pediatric hospitals contributed 241 eligible patients administered oral azithromycin and 367 patients administered IV/IM fentanyl (Tables 1, 2, 4).

\subsection{Exposure to Oral Azithromycin and Serious Adverse Events}

Of the 241 children exposed to oral azithromycin, 106 (44\%) were female, while the median patient age was 6 years (range $0-20)$ and the most commonly reported race was black (100, $42 \%)$. The most common comorbidities were those affecting the respiratory system $(121,50 \%)$, congenital/chromosomal disorders $(63,26 \%)$, and diseases of blood and immune disorders $(47,20 \%)$. There were 17 patients in the azithromycin cohort who were 16-20 years of age. Only three of these 17 adolescents had an SAE.

The median maximum daily dose for azithromycin was $10 \mathrm{mg} / \mathrm{kg}$ (range 2.2-21.8), with the median cumulative dose at $25 \mathrm{mg} / \mathrm{kg}$ (range $4-170.5$ ) (Table 2).

Ninety-three patients (39\%) in the azithromycin cohort were administered the drug off-label, with most off-label use being by indication only $(65,27 \%$ of all patients), but the specific indications for off-label use were not determined. A total of 21 patients (9\%) experienced at least one of the identified 32 SAEs. Thirteen patients that were on-label experienced an SAE and eight that were off-label. Prolonged diarrhea/loose stool, noted in six individuals ( $2 \%$ of the cohort) was the most common SAE (Table 2). All of the patients with diarrhea on azithromycin were taking the drug on-label.

For the azithromycin regression analysis, five confounders were deemed eligible: sex, Asian race/ethnicity, total comorbidities, having a blood or immune system related comorbidity, and total concomitant medications. The logistical regression model included 235 individuals with complete data once the confounders were included. Off-label use was not a significant predictor of SAEs $(p=0.81)$, though females, Asian patients, and the number of concomitant medications administered to the patient were associated significantly with SAE risk (Table 3).

The sensitivity analysis showed that when the correlation coefficient cut-off was reduced from $|r| \geq 0.1$ to $|r| \geq 0.05$ for eligible confounders, the azithromycin regression model included six additional confounders: black and
Hispanic race/ethnicity, maximum daily dose, cumulative dose, specific comorbidities (infectious and parasitic disease, congenital malformations and chromosomal mutations) (ESM Table 1). However, off-label use of oral azithromycin was still not significantly associated with risk of any SAE $(p=0.83)$.

A Kaplan-Meier plot was generated to characterize how the risk of any SAE changed over the course of therapy with azithromycin (ESM Fig. 1). In addition, a Cox proportional hazards regression was used to compare time to event (first SAE reported) in on-label compared with off-label groups. The Cox proportional hazards assumption was fulfilled for the analysis $(p=0.90)$. In this regression, the off-label group was associated with reduced risk of any SAE, but this was not statistically significant [hazard ratio (HR) $0.83,95 \%$ CI $0.31-2.23 ; p=0.71]$. Female and Asian patients as well as the number of concomitant medications administered to the patient were still associated significantly with SAE risk.

\subsection{Exposure to IV/IM Fentanyl and Serious Adverse Events}

Of the 367 children exposed to fentanyl, 149 were female (41\%), while median patient age was 2 months (range $0-18$ years) and the most commonly reported race/ethnicity was white $(134,37 \%)$. The most common comorbidities were congenital/chromosomal disorders $(142,39 \%)$, followed by those affecting the circulatory system $(120,33 \%)$ and respiratory system $(101,28 \%)$. There were 16 patients in the fentanyl cohort who were 16-20 years of age. Only two of 16 16- to 20-year-olds had an SAE. The median maximum daily dose for fentanyl excluding patients with PRN dosing was $2.1 \mathrm{mcg} / \mathrm{kg}(n=211$, range $0.1-156)$, and the median cumulative dose over the entire hospitalization was $2.0 \mathrm{mcg} / \mathrm{kg}(n=180$, range $0.1-1884)$ (Table 4). Patients in this study had a variety of dosing regimens, including continuous infusion, single-dose bolus, and doses given at different times. These were standardized into a cumulative dose given for the day and then expanded to a cumulative dose per hospital stay. The sample size in this metric is lower because it excludes patients with dosing recorded as PRN at some point during hospitalization, with the exact amount administered not recorded. There were also four patients where fentanyl dosing was not adequately reported, so we were unable to calculate a weight-based dose.

It is important to note that fentanyl dosing regimens included a mix of continuous infusions, boluses, and single injections. One patient may have received all three types of administration during their hospitalization. Patients in our fentanyl cohort had up to eight different regimens during hospitalization, though it should be noted only three patients had four or more different regimens. There are also some 
Table 2 Summary data for azithromycin cohort

\begin{tabular}{|c|c|c|}
\hline Variable & $N$ with non-missing & $\begin{array}{l}\text { Azithromy- } \\
\text { cin exposed } \\
(N=243)^{\mathrm{a}}\end{array}$ \\
\hline Age (years) & 241 & $6.25(0-20)$ \\
\hline Weight (kg) & 237 & $23.5(2.3-142)$ \\
\hline Sex (female) & 240 & $106(44 \%)$ \\
\hline Race & 236 & \\
\hline Black & & $100(42 \%)$ \\
\hline White & & $57(24 \%)$ \\
\hline Hispanic & & $45(19 \%)$ \\
\hline Asian & & $9(4 \%)$ \\
\hline Other & & $25(11 \%)$ \\
\hline Site of admittance ${ }^{\mathrm{b}}$ & 241 & \\
\hline PICU & & $210(87 \%)$ \\
\hline NICU & & $8(3 \%)$ \\
\hline Other site & & $26(11 \%)$ \\
\hline Hospital length of stay (days) & 231 & $5(1-224)$ \\
\hline Comorbidities $^{\mathrm{b}}$ & 241 & $1(0-14)$ \\
\hline Respiratory system & & $121(50 \%)$ \\
\hline Congenital/chromosomal & & $63(26 \%)$ \\
\hline Diseases of blood and immune disorders & & $47(20 \%)$ \\
\hline Circulatory system & & $34(14 \%)$ \\
\hline Digestive system & & $26(11 \%)$ \\
\hline Nervous system & & $25(10 \%)$ \\
\hline Infectious/parasitic diseases & & $23(10 \%)$ \\
\hline Total concomitant medications & 241 & $5(0-20)$ \\
\hline Median maximum daily azithromycin dose $(\mathrm{mg} / \mathrm{kg})$ & 240 & $10(2.2-21.8)$ \\
\hline Median cumulative azithromycin dose (mg/kg) & 240 & $25(4-170.5)$ \\
\hline Off-label use & 241 & \\
\hline Off-label use by either age or indication & & $93(39 \%)$ \\
\hline Off-label use by age only ( $<6$ months) & & $6(2 \%)$ \\
\hline Off-label use by indication only & & $65(27 \%)$ \\
\hline Off-label use by both age and indication & & $22(9 \%)$ \\
\hline SAE reported & 241 & $0(0-5)$ \\
\hline Patients with any $\mathrm{SAE}^{\mathrm{b}}$ & & $21(9 \%)$ \\
\hline Diarrhea/Loose Stool & & $6(2 \%)$ \\
\hline Hypersensitivity & & $5(2 \%)$ \\
\hline Vomiting & & $4(2 \%)$ \\
\hline Rash & & $3(1 \%)$ \\
\hline Nausea & & $3(1 \%)$ \\
\hline Death & & $2(1 \%)$ \\
\hline Other ${ }^{c}$ & & $9(4 \%)$ \\
\hline
\end{tabular}

$P I C U$ pediatric intensive care unit, NICU neonatal intensive care unit, SAE serious adverse event

${ }^{a}$ Data are shown as median (range) for continuous variables and $N(\%)$ for categorical variables

${ }^{\mathrm{b}}$ Patients may have more than one

${ }^{c} 9$ patients accounted for one instance each of desaturations, finger discoloration, hepatomegaly, hepatotoxicity, hyperglycemia, lip swelling, nephrotic syndrome, neutropenia, and seizure 
Table 3 The effect of off-label use of oral azithromycin on the risk of patient SAE $(N=235)$

\begin{tabular}{|c|c|c|c|}
\hline Variable & Odds ratio & $p>|z|$ & $95 \%$ conf. interval \\
\hline \multicolumn{4}{|c|}{ Dependent variable: any SAE reported during azithromycin administration with 24-day lag for metabolic clearance, $[0,1]$ binary } \\
\hline \multicolumn{4}{|l|}{ Primary independent variables } \\
\hline $\begin{array}{l}\text { Off-label use by either age group or indication } n=93 \text { in this group, } 8 \text { had any } \\
\text { SAE }\end{array}$ & 0.87 & 0.808 & $0.27-2.71$ \\
\hline \multicolumn{4}{|l|}{ Confounders } \\
\hline Female & - & $0.045^{*}$ & - \\
\hline Asian & - & $0.009 * *$ & - \\
\hline $\ln ($ total comorbidities) & - & 0.472 & - \\
\hline Specific comorbidity & & & - \\
\hline Diseases of the blood and blood-forming organs, immune disorders & - & 0.084 & \\
\hline $\ln ($ total concomitant medications) & - & $0.000 * *$ & - \\
\hline Wald $\chi^{2}$ & 23.78 & $0.000 * *$ & \\
\hline Pseudo $R^{2}$ & 0.22 & & \\
\hline
\end{tabular}

Logistical regression (logit) with Odds Ratios reported and Robust Standard Errors. Denominator is not $N=241$ as one patient had a missing value for sex and five additional patients did not have race or ethnic data

SAE serious adverse event

*Significant at the $95 \%$ confidence level; **Significant at the $99 \%$ confidence level

outlier cumulative doses for fentanyl. For instance, nine patients had a cumulative dose of over $100 \mathrm{mcg} / \mathrm{kg}$ per stay, with three of these patients administered over $500 \mathrm{mcg} / \mathrm{kg}$. The highest recorded was a cumulative dose of $1884 \mathrm{mcg} / \mathrm{kg}$ over the course of a 52-day hospital stay (24 days of fentanyl administered). This patient was administered a changing regimen of fentanyl continuous infusion with $108 \mathrm{mcg} / \mathrm{kg} /$ day at the highest level. This patient had no SAEs reported.

Off-label use of fentanyl $(287,78 \%)$ was predominant and involved mostly off-label use by both age and indication (129, 35\% of all patients) and off-label use by age only (131, 36\% of all patients). Of the 367 children exposed to fentanyl, a total of 95 patients (26\%) (15 on-label and 80 off-label) experienced at least one SAE. The most common SAEs were respiratory depression $(37,10 \%)$ (ventilator use not captured in this study), death (30,8\%), and circulatory depression $(29,8 \%$ ) (Table 4). Only two patients who had respiratory depression were on-label, while 35 patients who had respiratory depression were off-label.

For the logistic regression, five variables were identified as eligible confounders for the outcome of any SAE reported with exposure to fentanyl: total comorbidities, specific comorbidities (nervous system disease, respiratory disease, and perinatal disease), and total concomitant medications. Off-label use of fentanyl by both age and indication was associated with increased risk for any SAE, but was not significant (OR 1.99; 95\% CI 0.94-4.19; $p=0.07$ ) (Table 5). A sub-analysis for the association of respiratory depression with off-label use of fentanyl included two eligible confounders: respiratory disease and perinatal disease.
Adjusting for these confounders, off-label use of fentanyl by both age and indication was associated with a higher risk of respiratory depression $(n=24$; OR 5.05 ; 95\% CI $1.08-23.56$, $p=0.04$ ) (Table 5).

For our first sensitivity analysis, the criterion for eligible confounders was broadened from $|r| \geq 0.1$ to $|r| \geq 0.05$. The association of off-label use of fentanyl by both age and indication with any SAE increased significantly (OR 2.53; 95\% CI $1.13-5.65 ; p=0.02$ ). When the eligible confounders were broadened for the outcome of respiratory depression only, offlabel use of fentanyl by both age and indication (OR 5.05; 95\% CI 1.05-24.27; $p=0.04$ ) remained significant (ESM Table 2).

When the dosing variables were included in the fentanyl regression model, the sample size decreased to 178 as patients with any PRN dosing were excluded from the analysis. Only 180 patients had complete drug regimen information that did not include any PRN dosing and an additional six patients were missing race/ethnicity, with an overlap of four with the group above. In this analysis, off-label use of fentanyl by both age and indication $(n=41)$ was not significantly associated with the risk of any SAE (OR 3.38; 95\% CI $0.93-12.28 ; p=0.06$ ). In addition, cumulative dose was not associated with the risk of any SAE (ESM Table 3).

Kaplan-Meier plots were generated to characterize how the risk of any SAE or the risk of respiratory depression by itself changed over the course of therapy with fentanyl, with a focus on off-label use by both age and indication (Fig. 1, ESM Fig. 2). Cox proportional hazards regression was used to compare time to event (first SAE reported or respiratory depression reported) in on-label compared with off-label 
Table 4 Summary data for fentanyl cohort

\begin{tabular}{|c|c|c|}
\hline Variable & $N$ with non-missing & Fentanyl exposed $(N=374)^{2}$ \\
\hline Age & 367 & 2 months $(0-18$ years $)$ \\
\hline Weight (kg) & 365 & $3.8(0.4-100)$ \\
\hline Sex (female) & 361 & $149(41 \%)$ \\
\hline Race & 361 & \\
\hline Black & & $70(19 \%)$ \\
\hline White & & $130(36 \%)$ \\
\hline Hispanic & & $97(27 \%)$ \\
\hline Asian & & $21(6 \%)$ \\
\hline Other race & & $43(12 \%)$ \\
\hline Site of Admittance ${ }^{\mathrm{b}}$ & 367 & \\
\hline PICU & & $144(40 \%)$ \\
\hline NICU & & $189(52 \%)$ \\
\hline Other site & & $33(9 \%)$ \\
\hline Hospital length of stay (days) & 336 & $17(1-213)$ \\
\hline Comorbidities $^{\mathrm{b}}$ & 367 & $2(0-11)$ \\
\hline Congenital/chromosomal & & $142(39 \%)$ \\
\hline Circulatory system & & $120(32 \%)$ \\
\hline Respiratory system & & $101(28 \%)$ \\
\hline Perinatal period & & $88(24 \%)$ \\
\hline Digestive system & & $83(23 \%)$ \\
\hline Nervous system & & $75(20 \%)$ \\
\hline Total concomitant medications & 367 & $5(0-20)$ \\
\hline Median maximum daily fentanyl dose $(\mathrm{mcg} / \mathrm{kg})^{\mathrm{c}}$ & 211 & $2.1(0.1-156)$ \\
\hline Median cumulative fentanyl dose $(\mathrm{mcg} / \mathrm{kg})^{\mathrm{A}}$ & 180 & $2.0(0.1-1884)$ \\
\hline Off-label use & 367 & \\
\hline Off-label use by either age or indication & & $287(78 \%)$ \\
\hline Off-label use by age only ( $<2$ years) & & $131(36 \%)$ \\
\hline Off-label use by indication only & & $27(7 \%)$ \\
\hline Off-label use by both age and indication & & $129(35 \%)$ \\
\hline SAE reported & 367 & $0(0-5)$ \\
\hline Patients with any $\mathrm{SAE}^{\mathrm{b}}$ & & $95(26 \%)$ \\
\hline Respiratory depression & & $37(10 \%)$ \\
\hline Death & & $30(8 \%)$ \\
\hline Circulatory depression & & $29(8 \%)$ \\
\hline Apnea & & $14(4 \%)$ \\
\hline Bradycardia $^{c}$ & & $16(4 \%)$ \\
\hline Respiratory arrest & & $4(1 \%)$ \\
\hline Acidosis & & $4(1 \%)$ \\
\hline Cardiac arrest & & $3(1 \%)$ \\
\hline Desaturation & & $3(1 \%)$ \\
\hline Other $^{\mathrm{d}}$ & & $20(5 \%)$ \\
\hline
\end{tabular}

$P I C U$ pediatric intensive care unit, NICU neonatal intensive care unit, SAE serious adverse event ( ${ }^{\mathrm{A}}$ excludes patients that received any PRN dosing)

${ }^{a}$ Data are shown as median (range) for continuous variables and $N(\%)$ for categorical variables

${ }^{\mathrm{b}}$ Patients may have more than one

${ }^{\mathrm{c}}$ One patient had a second episode of bradycardia within $24 \mathrm{~h}$, but is not double counted here

${ }^{\mathrm{d}} 20$ patients accounted for 22 other SAEs including hypotension (2), seizure (2), sepsis (2), and one instance each of carbon dioxide retention after placing peripherally inserted central catheter, chest tightness, constipation, extracorporeal membrane oxygenation requirement, hypertension, intestinal perforation, large hemorrhagic bleed, myoclonic jerks in extremities, naloxone requirement likely due to morphine, necrotizing enterocolitis, neutropenia, pneumothorax, pulmonary edema, reflux with aspiration, respiratory alkalosis, and tachycardia 
Table 5 The effect of off-label use of IV/IM fentanyl on the risk of any patient SAE and the risk of respiratory depression $(N=367)$

\begin{tabular}{|c|c|c|c|}
\hline Variable & Odds ratio & $p>|z|$ & $95 \%$ conf. interval \\
\hline \multicolumn{4}{|c|}{ Dependent variable: any SAE reported during fentanyl administration with 2-day lag for metabolic clearance, $[0,1]$ binary } \\
\hline \multicolumn{4}{|c|}{ Primary independent variables } \\
\hline Off-label use by age group only, $<2$ years $n=131$ in this group, 28 had any SAE & 1.36 & 0.429 & $0.63-2.95$ \\
\hline Off-label use by indication only $n=27$ in this group, 4 had any SAE & 0.52 & 0.295 & $0.15-1.77$ \\
\hline Off-label use by both age and indication $n=129$ in this group, 48 had any SAE & 1.99 & 0.070 & $0.94-4.19$ \\
\hline \multicolumn{4}{|l|}{ Confounders } \\
\hline $\ln ($ total comorbidities $)$ & - & 0.412 & - \\
\hline \multicolumn{4}{|l|}{ Specific comorbidity } \\
\hline Diseases of the nervous system & - & $0.042^{*}$ & - \\
\hline Diseases of the respiratory system & - & 0.472 & - \\
\hline Diseases of the perinatal period & - & 0.064 & - \\
\hline $\ln ($ total concomitant medications) & - & $0.002 * *$ & - \\
\hline Wald $\chi^{2}$ & 30.21 & $0.000 * *$ & \\
\hline Pseudo $R^{2}$ & 0.09 & & \\
\hline \multicolumn{4}{|c|}{ Dependent variable: respiratory depression reported during fentanyl administration with 2-day lag for metabolic clearance, $[0,1]$ binary } \\
\hline \multicolumn{4}{|l|}{ Primary independent variables } \\
\hline Off-label use by age group only, $<2$ years $n=131$ in this group, 9 had RD & 1.97 & 0.402 & $0.40-9.64$ \\
\hline Off-label use by indication only $n=27$ in this group, 2 had RD & 2.52 & 0.386 & $0.31-20.44$ \\
\hline Off-label use by both age and indication $n=129$ in this group, 24 had RD & 5.05 & $0.040^{*}$ & $1.08-23.56$ \\
\hline \multicolumn{4}{|l|}{ Confounders } \\
\hline \multicolumn{4}{|l|}{ Specific comorbidity } \\
\hline Diseases of the respiratory system & - & $0.023^{*}$ & - \\
\hline Diseases of the perinatal period & - & 0.057 & - \\
\hline Wald $\chi^{2}$ & 30.20 & $0.000 * *$ & \\
\hline Pseudo $R^{2}$ & 0.12 & & \\
\hline
\end{tabular}

Logistical regressions (logit) with odds ratios reported and robust standard errors

$R D$ respiratory depression, $S A E$ serious adverse event

*Significant at the $95 \%$ confidence level; **Significant at the $99 \%$ confidence level

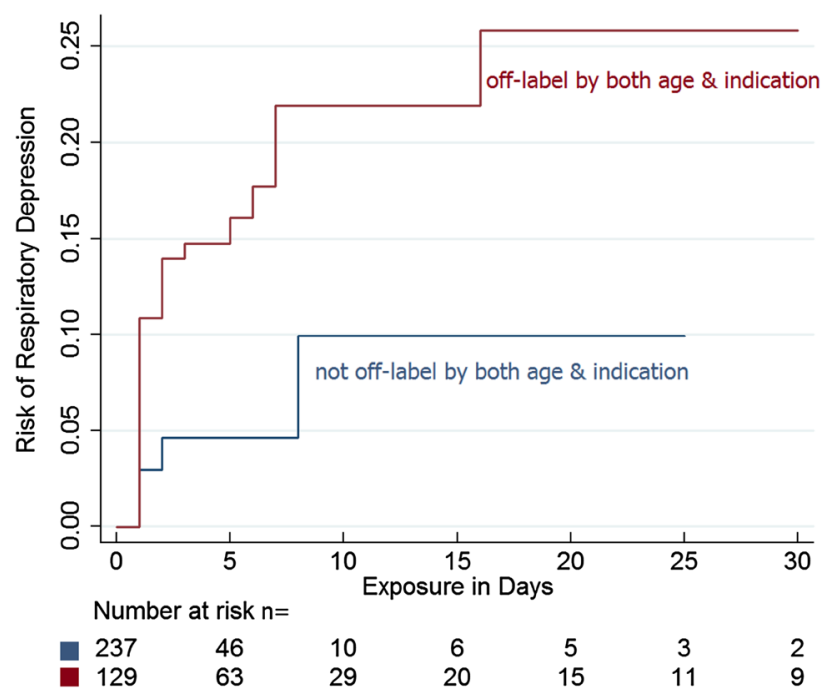

Fig. 1 Kaplan-Meier failure rate (risk of respiratory depression over time) for IV/IM fentanyl groups. The Cox proportional hazards assumption was fulfilled for the analysis of any $\operatorname{SAE}(p=0.31)$ and respiratory depression alone $(p=0.35)$. For the risk of any SAE over time, off-label use by age only (HR 1.10; 95\% CI 0.56-2.14; $p=0.78$ ), indication only (HR 0.54 ; 95\% CI $0.18-1.66$; $p=0.29$ ), and off-label by both age and indication (HR 1.28; 95\% CI 0.67-2.45; $p=0.46$ ) were all not significant. For the risk of respiratory depression over time, off-label use by age only (HR $1.85 ; 95 \%$ CI $0.39-8.79 ; p=0.44$ ), indication only (HR $1.13 ; 95 \%$ CI $0.10-12.65 ; p=0.92$ ), and off-label by both age and indication (HR 3.64; 95\% CI 0.81-16.42; $p=0.09$ ) were also not significant.

\section{Discussion}

For those children exposed to azithromycin during ICU admission, off-label use was less common than on-label use. Off-label use of azithromycin was not associated with higher rates of SAEs compared to on-label use. In contrast, off-label 
use of fentanyl was more common than on-label use in our cohort. Off-label use of fentanyl by both age and indication was associated with the SAE respiratory depression. The sensitivity analysis when more confounders were included in the regression equation showed the OR for the association between any SAE and off-label use of fentanyl was greater (OR 2.53, $p=0.02$ ) than that in the primary analysis (OR $1.99, p=0.07$ ). The statistical association was, however, not found for off-label use of fentanyl by indication only or by age only. The total number of concomitant medications and nervous system disease were significant confounders for the risk of SAEs with off-label use of fentanyl in our cohort. The confounding effect of total concomitant medications is a similar finding to that of earlier studies where polypharmacy was associated with an increased risk for ADEs in children [20-22]. To summarize, our analysis of fentanyl and azithromycin shows that off-label use may be more frequently associated with at least some SAEs in fentanyl-exposed patients, but not in azithromycin-exposed patients. Thus, the nature of these outcomes was drug dependent in this study.

Czaja et al. [23] found azithromycin as the most frequently used antimicrobial agent among PICU admissions in 39 children's hospitals across the USA. In the Czaja study, of the 4683 children who received azithromycin, $92 \%$ were given the drug off-label. In comparison, 39\% of patients in our study were administered oral azithromycin off-label. The difference in off-label rates may be due to differences in study design, as the prior study used the PHIS database, which is a database that includes encounter and diagnosis codes, whereas we performed manual review of each record. The Czaja study did not report individual SAEs associated with azithromycin, so event rates across studies cannot be compared.

It is also worth noting that azithromycin is not on the Institute for Safe Medication Practices (ISMP) [24] list of high-alert medications and was not categorized as a highrisk medicine in a previous study evaluating its off-label use in PICUs [23]. Furthermore, the sensitivity analysis for azithromycin indicated that, in the presence of 11 confounders, off-label by age or indication was not significantly associated with an increased risk of SAEs.

These prior results taken together with our study findings suggest that off-label use of azithromycin does not appear to pose a clinically significant elevated risk to children compared to on-label use. However, the FDA (including the BPCA program) has since included azithromycin on highpriority lists because of the lack of pediatric safety data [23, 25]. This high-priority status was based on drugs with a written request from the FDA to the manufacturer for pediatric studies or those drugs that exist on the BPCA therapeutic priority list for further studies of their safety in children. It is hoped that our finding of a lack of a consistent association between off-label azithromycin use and SAEs would add to the evidence of azithromycin safety in children.

In our study, off-label use of fentanyl was predominant, which is consistent with previous publications. Other surveys have shown that fentanyl is one of the most commonly used sedative and analgesic agents for children admitted to the ICU in the USA $[23,26]$. We identified off-label use of fentanyl by age with or without indication in 260 fentanyl-exposed children (71\%), a combination of the mutually exclusive groups of off-label by age only $(131,36 \%)$ and offlabel by both age and indication $(129,35 \%)$. This is similar to the rate of off-label use by age of $88 \%$ in a Canadian study [17]. A previous multicenter study in the USA documented $44 \%$ off-label use by age or indication for fentanyl among PICU admissions [23] versus our study's 78\% off-label use by age or indication. This variation in the rate of off-label fentanyl use could be attributed to the differences in setting, sample size, group of patients studied, the duration of fentanyl exposure, or the specification of route of administration. In addition, we evaluated SAEs associated with off-label IV/IM fentanyl use only, while other studies focused on offlabel use of fentanyl and other drugs.

When fentanyl was administered off-label by both age and indication, this combination was more likely to be associated with SAEs than off-label use by age only. We found no literature on the association between off-label drug use for children and the risk of SAEs, as defined above. However, Horen et al. reported that usage which did not have the FDA-approved pediatric indication was associated with an increased risk of adverse drug reactions-defined differently from SAE - among pediatric outpatients, particularly infants [27]. In our study, fentanyl patients needed to be treated both off-label by age and indication to show an elevated risk of at least one SAE. It may be that each risk factor alone is insufficient to increase risk, but that together there may be an additive risk of off-label use. Our data suggest that at least some SAEs associated with fentanyl use in the pediatric ICU setting may be influenced by the manner of its off-label use.

Off-label use of fentanyl by both age and indication in our cohort is associated with a 5.05 times greater likelihood of respiratory depression. Respiratory depression is a major adverse event associated with opioid use, including fentanyl [28]. Two life-threatening AEs (hypoventilation and hypotension) were reported among hospitalized children on opioids in a multicenter cohort study involving five countries across Australia, Europe and Asia [29]. One possibility regarding the apparent association of off-label use with respiratory depression is that fentanyl has a narrow therapeutic index. A retrospective study evaluating the AEs of a continuous infusion of fentanyl for postoperative pain relief in 1166 children aged 0-14 years in Japan reported respiratory depression as a common SAE in $0.8 \%$ of the cohort 
[30]. This low rate, compared to the $16 \%$ of patients with respiratory depression observed in our study, may be attributed to several factors including differences in the age of the patients and the outcomes measured. In addition, included in our population were those who experienced a range of severe respiratory depression (breathing devices were not specified in this study), while the Japanese study focused on children $\leq 14$ years with severe respiratory depression for which mask ventilation or tracheal intubation was required. However, the determinants of the risk for this important SAE were not evaluated in the Japanese study. Since serious, lifethreatening, or fatal respiratory depression may occur with the use of fentanyl injection, the FDA has included a boxed warning to monitor for respiratory depression with fentanyl use, especially during initiation of the medicine or following a dose increase [12].

Our study had a number of limitations. First, it was a retrospective study with a convenience sample. It is rather complex to accurately determine the occurrence of SAEs in an ICU setting since the symptoms of the event may overlap with an underlying disease, comorbidity, and adverse events from concomitant medication or may be caused by several unrelated factors, including the pharmacokinetic profile of the drug. There is also the potential for misclassification of on- versus off-label use, specifically if the appropriate indication for azithromycin use was present but not clearly documented in the EHR. These factors could more easily be captured in a prospective study with comparator groups. In addition, a prospective design would enable complete ascertainment of consecutive drugexposed patients, which would reduce the risk of selection bias due to exclusion of individuals without sufficient data to determine SAE outcomes.

In addition, the two drugs chosen differed in many ways such as therapeutic class, route of administration, and therapeutic margin for safety. Furthermore, the offand on-label groups of patients may have differed in many ways, including the extent of illness (proxies were included in our regressions), the presence and number of concomitant medications (included in our regressions), and the proximity in time to surgical procedures (included in our case definition). Since the indications for off-label use were not systematically collected, this variable was not assessed. Some markers of severe illness could not be detected, such as respiratory depression in ventilated patients. Although our regression models accounted for the important confounders that we identified, they clearly did not include all.

This study was also limited by incomplete data collection. Although dose was included as a possible confounder in the case of azithromycin, in the case of fentanyl, only a sensitivity analysis based on approximately half of the population could be conducted due to missing data from regimens that included PRN dosing. For patients without PRN dosing, there were assumptions made in the calculation of continuous infusion data that in some cases could have over- or under-estimated the exact dose administered. However, the dosing information we did capture in a subset of fentanyl-exposed patients did not suggest that dose was a confounder. Potential covariates that were not analyzed as part of this study but are potential subjects of future research include the impact of disease severity scores, mechanical ventilation, and indication for ICU admission. Inclusion of doses outside the recommended range in the definition of "off-label" would be an area of further research. Another potential area of future research is the incidence and risk factors for SAEs with longer latency.

\section{Conclusion}

In the setting of pediatric ICUs, off-label use of fentanyl appears to be associated with an increased risk of respiratory depression compared to on-label use when the drug is administered to a patient simultaneously for an unlabeled age group and unlabeled indication. Off-label use of azithromycin in this setting appears not to be associated with an increased risk of SAEs compared to on-label use. Prospective studies should be undertaken to assess the safety and efficacy of fentanyl in the pediatric population so that data can be added to the FDA labeling.

Acknowledgements We thank Drs. Mark Levenson and Elande Baro for their guidance with the statistical analysis on this project. This work was supported by UL1 TR000445 to Vanderbilt University Medical Center, T32 GM007569 (KAO) and Burroughs Wellcome Fund IRSA 1015006 (SLV).

\section{Compliance with Ethical Standards}

Kazeem A. Oshikoya, Gerold T. Wharton, Debbie Avant, Norman E. Fenn, Allison Lardieri, Edwin Doe, Beena G. Sood, Carol Taketomo, Phuong Lieu, Lilly Yen, and Ann W. McMahon have no conflicts of interest. Sara L. Van Driest has been an invited speaker to Merck and received an honorarium. No additional funding was provided for this study. The opinions expressed in this article are those of the authors and should not be interpreted as the position of the US Food and Drug Administration.

\section{References}

1. Sachs A, Avant D, Lee C, Rodriguez W, Murphy D. Research letter: pediatric information in drug product labeling. JAMA. 2012;307(18):1914-5.

2. Ufer M, Kimland E, Bergman U. Adverse drug reactions and offlabel prescribing for paediatric outpatients: a one-year survey of spontaneous reports in Sweden. Pharmacoepidemiol Drug Saf. 2004;13:147-52. 
3. Bellis JR, Kirkham JJ, Nunn AJ, Pirmohamed M. Adverse drug reactions and off-label and unlicensed medicines in children: a prospective cohort study of unplanned admissions to a paediatric hospital. Br J Clin Pharmacol. 2014;77:545-53.

4. Aagaard L. Off-label and unlicensed prescribing of medicines in pediatric populations: occurrence and safety aspects. Basic Clin Pharmacol Toxicol. 2015;117(4):215-8.

5. Food and Drug Administration, HHS. 21 CFR 314.80: Post marketing reporting of adverse drug experiences 2017. https://www. gpo.gov/fdsys/pkg/CFR-2011-title21-vol5/pdf/CFR-2011-title 21-vol5-sec314-80.pdf. Accessed 30 Jul 2017.

6. Bellis JR, Kirkham JJ, Thiesen S, Conroy EJ, Bracken LE, Mannix HL, et al. Adverse drug reactions and off-label and unlicensed medicines in children: a nested case-control study of inpatients in a pediatric hospital. BMC Med. 2013;7(11):238. https://doi. org/10.1186/1741-7015-11-238.

7. Alamu JO. Evaluation of antimicrobial use in a pediatric intensive care unit. Ph.D. (Doctor of Philosophy) thesis, University of Iowa. 2009. http://ir.uiowa.edu/etd/277. Accessed 06 Jun 2017.

8. Tobias JD. Sedation in the intensive care unit: challenges, outcomes, and failure strategies. In: Manson KP, editor. Pediatric sedation outside of the operating room: a multispecialty international collaboration. New York: Springer; 2015. p. 125-49.

9. Shah SS, Hall M, Goodman DM, Feuer P, Sharma V, Fargason C Jr, et al. Off-label drug use in hospitalized children. Arch Pediatr Adolesc Med. 2007;161(3):282-90.

10. Zithromax (azithromycin) [package insert]. Pfizer, Inc. New York, NY; March 30, 2017. https://www.accessdata.fda.gov/drugsatfda _docs/label/2017/050670s032,050710s046,050711s043,05078 4s030lbl.pdf. Accessed 8 Jun 2017.

11. Tiwari T, Murphy TV, Moran J. Recommended antimicrobial agents for the treatment and post-exposure prophylaxis of pertussis: 2005 CDC Guidelines. MMWR Recomm Rep. 2005;54(RR-14):1-16.

12. Fentanyl citrate [package insert]. Hospira Inc. Pfizer Inc. Lake Forest, IL; December 2016. https://www.accessdata.fda.gov/drugsatfda _docs/label/2016/019115s030s031lbl.pdf. Accessed 8 Jun 2017.

13. Prescilla RP. The pharmacology and clinical application of sedatives, analgesics, and adjuncts. In: Manson KP, editor. Pediatric sedation outside of the operating room: a multispecialty international collaboration. New York: Springer; 2015. p. 125-49.

14. Corny J, Bailey B, Lebel D, Bussières JF. Unlicensed and off-label drug use in paediatrics in a mother-child tertiary care hospital. Paediatr Child Health. 2016;21(2):83-7.

15. Institute for Safe Medication Practices. QuarterWatch (special report, 2014): Adverse drug events in children less than 18 years old. 2017. https://www.ismp.org/newsletters/acutecare/showarticl e.aspx?id=67. Accessed 01 Aug 2017.

16. Silva DCB, Araujo OR, Arduini RG, Alonso CFR, Shibata ARO, Troster EJ. Adverse drug events in a paediatric intensive care unit: a prospective cohort. BMJ Open. 2013;3(2):e001868. https://doi. org/10.1136/bmjopen-2012-001868.

17. Agarwal S, Classen D, Larsen G, Tofil NM, Hayes LW, Sullivan JE, et al. Prevalence of adverse events in pediatric intensive care units in the United States. Pediatr Crit Care Med. 2010;11(5):568 78. https://doi.org/10.1097/PCC.0b013e3181d8e405.

18. U.S. Food and Drug Administration. CFR - Code of Federal Regulations Title 21. 2017. https://www.accessdata.fda.gov/scripts/cdrh/ cfdocs/cfCFR/CFRSearch.cfm?fr=201.57. Accessed 30 Dec 2017.

19. Brunton LL, Knollmann BC, Hilal-Dandan R. Goodman and Gilman's the pharmacological basis of therapeutics. 13th ed. New York: McGraw Hill Medical; 2018.

20. Khan LM, Al-Harthi SE, Saadah OI. Adverse drug reactions in hospitalized pediatric patients of Saudi Arabian university hospital and impact of pharmacovigilance in reporting ADR. Saudi Pharm J. 2013;21(3):261-6.

21. Priyadharsini R, Surendiran A, Adithan C, Sreenivasan S, Sahoo FK. A study of adverse drug reactions in pediatric patients. J Pharmacol Pharmacother. 2011;2(4):277-80.

22. Vázquez-Alvarez AO, Brennan-Bourdon LM, Rincón-Sánchez AR, Islas-Carbajal MC, Huerta-Olvera SG. Improved drug safety through intensive pharmacovigilance in hospitalized pediatric patients. BMC Pharmacol Toxicol. 2017;18(1):79.

23. Czaja AS, Reiter PD, Schultz ML, Valuck RJ. Patterns of off-label prescribing in the pediatric intensive care unit and prioritizing future research. J Pediatr Pharmacol Ther. 2015;20(3):186-96.

24. Institute for Safe Medication Practices List of High-Alert Medication. 2017. http://www.ismp.org/Tools/institutionalhighAlert.asp. Accessed 6 Jun 2017.

25. BPCA priority list of pediatric therapeutic needs. 2017. http://bpca. nichd.nih.gov/prioritization/status.cfm. Accessed 6 Jun 2017.

26. Rhoney DH, Murry KR. National survey on the use of sedatives and neuromuscular blocking agents in the pediatric intensive care unit. Pediatr Crit Care Med. 2002;3:129-33.

27. Horen B, Montastruc JL, Lapeyre-Mestre M. Adverse drug reactions and off-label drug use in pediatric outpatients. Br J Clin Pharmacol. 2002;54(6):665-70.

28. Gill AM, Cousins A, Nunn AJ, Choonara IA. Opiate-induced respiratory depression in pediatric patients. Ann Pharmacother. 1996;30(2):125-9.

29. Rashed AN, Wong IC, Cranswick N, Hefele B, Tomlin S, Jackman $\mathrm{J}$, et al. Adverse drug reactions in children-international surveillance and evaluation (ADVISE): a multicentre cohort study. Drug Saf. 2012;35(6):481-94.

30. Kabara S, Kagawa T, Ikejima N, Takatsuji S, Sueda A. Side effects of continuous fentanyl infusion for postoperative pain relief in children. Masui. 2015;64(8):799-803. 